\title{
RELATIVE NORMAL COMPLEMENTS IN FINITE GROUPS
}

\author{
PAMELA A. FERGUSON
}

\begin{abstract}
G, H, H_{0}, \pi\right)$ denotes the following configuration: $H$ and $H_{0}$ are the subgroups of the finite group $G$ with $H_{0} \unlhd H$ and $\pi$ is the set of primes dividing $\left(H: H_{0}\right)$. For $\left(G, H, H_{0}, \pi\right)$ we consider conditions $(\mathrm{A}),\left(\mathrm{B}_{0}\right)$, and $(\mathrm{C}):(\mathrm{A})$ Any two $\pi$-elements of $H-H_{0}$ which are $G$-conjugate are $H$-conjugate. $\left(\mathrm{B}_{0}\right)$ For each $\pi$-element $x \in H-H_{0}, C_{G}(x)=I(x) C_{H}(x)$ where $I(x)$ is a normal $\pi^{\prime}$-subgroup of $C_{G}(x)$. (C) $\left|\left(H-H_{0}\right)^{G, \pi}\right|=(G: H)\left|H-H_{0}\right|$. We show that if $\left(G, H, H_{0}, \pi\right)$ satisfies $\left(\mathrm{B}_{0}\right)$ and $(\mathrm{C})$, or $(\mathrm{A})$ and $\left(\mathrm{B}_{0}\right)$, and if $H / H_{0}$ is solvable, then there is a unique relative normal complement $G_{0}$ of $H$ over $H_{0}$.
\end{abstract}

All groups in this paper are finite. Given a group $G$ with subgroups $H_{0}, H$, and $G_{0}$ such that $H_{0} \unlhd H$, we call $G_{0}$ a relative normal complement of $H$ over $H_{0}$ if $G_{0} \unlhd G, G=G_{0} H$ and $H_{0}=G_{0} \cap H$.

Let $\pi(G)$ denote the set of primes dividing $|G|$. If $\pi$ is a set of primes, then the complementary set of primes will be denoted by $\pi^{\prime}$. A group $G$ is a $\pi$-group if $\pi(G) \subseteq \pi$. If $x \in G$, then $x$ is a $\pi$-element if $\langle x\rangle$ is a $\pi$-group. Every element $x$ of $G$ has a unique decomposition $x=x_{\pi} x_{\pi^{\prime}}=x_{\pi^{\prime}} x_{\pi}$ into a $\pi$-element $x_{\pi}$ and a $\pi^{\prime}$-element $x_{\pi^{\prime}}$. Further, $x_{\pi}$ and $x_{\pi^{\prime}}$ are powers of $x$. If $x$ and $y$ are elements of a subgroup $K$ of $G$, then $x$ and $y$ belong to the same $\pi$-section of $K$ if their $\pi$-parts $x_{\pi}$ and $y_{\pi}$ are $K$-conjugate. If $S$ is a subset of $G$, then $S^{G, \pi}$ denotes the union of all $\pi$-sections of $G$ which intersect $S$.

We let $\left(G, H, H_{0}, \pi\right)$ denote the following configuration: $G$ is a finite group with subgroups $H$ and $H_{0}$ such that $H_{0} \unlhd H$ and $\pi=\pi\left(H / H_{0}\right)$. For $\left(G, H, H_{0}, \pi\right)$ we consider the following conditions:

(A) Any two $\pi$-elements of $H-H_{0}$ which are $G$-conjugate are $H$-conjugate.

$\left(\mathrm{B}_{0}\right)$ For each $\pi$-element $x \in H-H_{0}, C_{G}(x)=I(x) C_{H}(x)$ where $I(x)$ is a normal $\pi^{\prime}$-subgroup of $C_{G}(x)$.

(C) $\left|\left(H-H_{0}\right)^{G, \pi}\right|=(G: H)\left|H-H_{0}\right|$.

Leonard [2] has shown that if $\left(G, H, H_{0}, \pi\right)$ satisfies conditions $\left(\mathrm{B}_{0}\right)$ and $(\mathrm{C})$ and $\pi=\{p\}$ or $I(x)$ is always a Hall $\pi^{\prime}$-subgroup of $C_{G}(x)$, then there is a unique relative normal complement $G_{0}$ of $H$ over $H_{0}$ and $G_{0}=G-\left(H-H_{0}\right)^{G, \pi}$. If $\left(G, H, H_{0}, \pi\right)$ satisfies conditions (A) and $\left(\mathrm{B}_{0}\right)$ and $\pi=\{p\}$, then Reynolds [3] has shown that there is a unique relative normal complement $G_{0}$ of $H$ over $H_{0}$ and $G_{0}=G-\left(H-H_{0}\right)^{G . \pi}$. In this paper, we prove two generalizations of these theorems.

Received by the editors January 13, 1982 and, in revised form, June 9, 1982.

1980 Mathematics Subject Classification. Primary 20E07. 
THEOREM 1. If $\left(G, H, H_{0}, \pi\right)$ satisfies conditions $\left(\mathrm{B}_{0}\right)$ and $(\mathrm{C})$ and $H / H_{0}$ is solvable, then there is a unique relative normal complement $G_{0}$ of $H$ over $H_{0}$ and $G_{0}=G-\left(H-H_{0}\right)^{G, \pi}$.

THEOREM 2. If $\left(G, H, H_{0}, \pi\right)$ satisfies conditions $(\mathrm{A})$ and $\left(\mathrm{B}_{0}\right)$ and $H / H_{0}$ is solvable, then there is a unique relative normal complement $G_{0}$ of $H$ over $H_{0}$ and $G_{0}=G-\left(H-H_{0}\right)^{G . \pi}$.

We omit stating explicitly the obvious corollaries which follow from Theorems 1 and 2 by replacing " $H / H_{0}$ is solvable" by " $\pi$ is a set of odd primes".

If a set $R$ is a disjoint union of $S$ and $T$, we write $R=S \dot{U} T$.

Lemma 1. Assume $\left(G, H, H_{0}, \pi\right)$ satisfies condition $\left(\mathrm{B}_{0}\right), K$ is a subgroup of $H$ containing $H_{0}$ and $\pi_{2} \subseteq \pi$. Then

$$
\left(K-H_{0}\right)^{G . \pi_{2}} \cup(H-K)^{G . \pi_{2}} \subseteq\left(H-H_{0}\right)^{G . \pi} .
$$

Proof. Assume $w \in(H-K)^{G . \pi_{2}} \cup\left(K-H_{0}\right)^{G . \pi_{2}}$, then $w=w_{\pi} w_{\pi^{\prime}}$ and $w_{\pi}=$ $w_{\pi_{2}} w_{\pi_{3}}$ where $\pi_{3}=\pi-\pi_{2}$. Further, $w_{\pi_{2}}=x^{g}$ where $x$ is a $\pi_{2}$-element in $H-K$ or $x$ is a $\pi_{2}$-element in $K-H_{0}$. Since $K \supseteq H_{0}, x$ is a $\pi$-element in $H-H_{0}$; hence $w^{g^{-1}} \in C_{G}(x)=I(x) C_{H}(x)$. Let $\tilde{w}=\left(w_{\pi}\right)^{g^{-1}}$, then $\tilde{w} I(x)=h I(x)$ where $h \in C_{H}(x)$. Since $I(x)$ is a normal $\pi^{\prime}$-subgroup of $C_{G}(x)$ and $\tilde{w}$ is a $\pi$-element, $h$ may be taken to be a $\pi$-element of $H$. Now $\langle\tilde{w}\rangle I(x)=\langle h\rangle I(x)$ and Theorem 6.2.1 of [1] imply that $\langle\tilde{w}\rangle=\langle h\rangle^{t}$ where $t \in I(x)$. It follows that $h^{i t}=\tilde{w}$ for some integer $i$, and $h_{\pi_{2}}^{i t}=\tilde{w}_{\pi_{2}}$ $=x$. Since $t \in C_{G}(x), h^{\prime}$ is a $\pi$-element of $H-H_{0}$ and $\tilde{w}_{\pi}$ is conjugate to an element of $H-H_{0}$. Thus, $w \in\left(H-H_{0}\right)^{G . \pi}$.

Proof of Theorem 1. If $\left(G, H, H_{0}, \pi\right)$ satisfies conditions $\left(\mathrm{B}_{0}\right)$ and $(\mathrm{C})$, then Leonard showed in $\S 3$ of [2], that $H-H_{0}$ is a union of $\pi$-sections of $H$ and condition (A) is satisfied.

Let $G$ be a minimal counterexample to Theorem 1. Theorem 1.2 of [2] implies that $\pi$ contains more than one prime. Since $H / H_{0}$ is solvable, there is a prime $p \in \pi$ such that $H$ has an abelian $p$ factor group. Thus, $H$ contains a normal subgroup $H_{1}$ of index $p$ and $H_{1} \supset H_{0}$. Let $D=H-H_{1}, x \in D$ and $y \in H$ where $x_{p}$ and $y_{p}$ are $H$-conjugate. Then $x_{p^{\prime}}$ and $y_{p^{\prime}} \in H_{1}$ and $x_{p} \in H-H_{1}$. Thus, $y_{p} \in H-H_{1}$ and $y \in H-H_{1}$. Therefore, $D$ is a union of $p$-sections of $H$. If $x$ and $y$ are $p$-elements in $D$ which are $G$-conjugate, then $x$ and $y$ are $G$-conjugate $\pi$-elements in $H-H_{0}$. Condition (A) implies $x$ and $y$ are $H$-conjugate. If $x$ is a $p$-element in $D$, then $C_{G}(x)=O_{p^{\prime}}\left(C_{G}(x)\right) C_{H}(x)$ follows from condition $\left(\mathrm{B}_{0}\right)$. Applying Theorem 2 of [3] to $G, H$, and $H_{1}$, we see that $G$ has a unique relative normal complement $G_{1}$ of $H$ over $H_{1}$ and $G_{1}=G-\left(H-H_{1}\right)^{G, p}$. Now $G=G_{1} \dot{\cup}\left(H-H_{1}\right)^{G, p}$ and $\left(G_{1}: H_{1}\right)=$ $(G: H)$ yield

$$
\left|\left(H-H_{1}\right)^{G . p}\right|=|G|-\left|G_{1}\right|=\left|G_{1}\right|\left(\left(H: H_{1}\right)-1\right)=\left(G_{1}: H_{1}\right)\left|H-H_{1}\right| .
$$

Let $R=G_{1} \cap\left(H-H_{0}\right)^{G \cdot \pi}$ and $\pi_{1}=\pi\left(H_{1} / H_{0}\right)$. We will show that $\left(G_{1}, H_{1}, H_{0}, \pi_{1}\right)$ satisfies the hypothesis of Theorem 1 and $R=\left(H_{1}-H_{0}\right)^{G_{1} \cdot \pi_{1}}$. It follows from Lemma $1, \pi_{1} \subseteq \pi$ and $H_{1} \supseteq H_{0}$, that $\left(H_{1}-H_{0}\right)^{G_{1} \cdot \pi_{1}} \subseteq R$. Let $w \in R$, then $w_{\pi}=x^{g}$ where $x$ is a $\pi$-element in $H-H_{0}$. We may write $g=h y$ where $h \in H$ 
and $y \in G_{1}$. Let $\tilde{w}=w_{\pi}^{y^{-1}}$ and $\tilde{x}=x^{h}$, then $\tilde{w} \in G_{1}$; hence, $\tilde{x} \in G_{1} \cap\left(H-H_{0}\right)=$ $H_{1}-H_{0}$. Since $y \in G_{1}$, it follows that $w \in\left(H_{1}-H_{0}\right)^{G_{1}, \pi_{1}}$ if $\pi_{1}=\pi$. If $\pi \neq \pi_{1}$, then $\tilde{x}=\tilde{x}_{\pi_{1}} \tilde{x}_{p}$. Now $H_{1} / H_{0}$ a $p^{\prime}$-group implies that $\tilde{x}_{p} \in H_{0}$. Thus, $\tilde{x}_{\pi_{1}} \in H_{1}-H_{0}$ and $\tilde{w}_{\pi_{1}}=\tilde{x}_{\pi_{1}}$. Again $y \in G_{1}$ so $w \in\left(H_{1}-H_{0}\right)^{G_{1} . \pi_{1}}$. Therefore, $R=\left(H_{1}-H_{0}\right)^{G_{1}, \pi_{1}}$. Lemma 1 , applied to $H_{1}$ and $p$, implies that $\left(H-H_{1}\right)^{G, p} \subseteq\left(H-H_{0}\right)^{G, \pi}$. Using $G=G_{1} \cup\left(H-H_{1}\right)^{G, p}$, we see that $\left(H-H_{0}\right)^{G, \pi}=R \cup\left(H-H_{1}\right)^{G, p}$. Now condition $(\mathrm{C})$ and $(G: H)=\left(G_{1}: H_{1}\right)$ yield

$$
\begin{aligned}
\left|\left(H_{1}-H_{0}\right)^{G_{1}, \pi_{1}}\right| & =|R|=\left|\left(H-H_{0}\right)^{G . \pi}\right|-\left|\left(H-H_{1}\right)^{G, p}\right| \\
& =\left(G_{1}: H_{1}\right)\left(\left|H-H_{0}\right|-\left|H-H_{1}\right|\right)=\left(G_{1}: H_{1}\right)\left|H_{1}-H_{0}\right| .
\end{aligned}
$$

Hence, $\left(G_{1}, H_{1}, H_{0}, \pi\right)$ satisfies condition (C).

Let $x$ be a $\pi_{1}$-element in $H_{1}-H_{0}$. Then $x$ is a $\pi$-element in $H-H_{0}$; hence, $C_{r_{1}}(x)=I(x) C_{\prime \prime}(x)$. Since $\pi_{1} \subseteq \pi$ and $\left(G: G_{1}\right)=p, I(x) \subseteq G_{1}$ and $I(x)$ is a normal $\pi_{1}^{\prime}$-subgroup of $C_{l_{j}}(x)$. Further, $C_{G_{1}}(x)=I(x) C_{H}(x) \cap G_{1}=I(x)\left(C_{H}(x) \cap G_{1}\right)=$ $I(x) C_{H_{1}}(x)$ so that condition $\left(\mathrm{B}_{0}\right)$ is satisfied by $\left(G_{1}, H_{1}, H_{0}, \pi_{1}\right)$.

Clearly $H_{1} / H_{0}$ is solvable since $H / H_{0}$ is. The minimality of $|G|$ now implies that $G_{1}$, has a unique relative normal complement $G_{0}$ of $H_{1}$ over $H_{0}$, namely $G_{0}=G_{1}-$ $\left(H_{1}-H_{0}\right)^{G_{1}, \pi_{1}}$. It has been shown that $\left(H_{1}-H_{0}\right)^{G_{1}, \pi_{1}}=R$ is a normal subset of $G$. Thus, $G_{0}$ is a normal subgroup of $G$. Clearly, $G_{0}$ is a relative normal complement of $H$ over $H_{0}$. Proposition 2.2 of [2] now implies that $G_{0}$ is unique and $G_{0}=G-$ $\left(H-H_{0}\right)^{G, \pi}$.

Proof of Theorem 2. Assume $\left(G, H, H_{0}, \pi\right)$ satisfies the hypothesis of Theorem 2 and let $D=H-H_{0}$. Assume $x \in D$ and $y \in H$ where $x_{\pi}$ is $H$-conjugate to $y_{\pi}$. Then $x_{\pi^{\prime}}$ and $y_{\pi^{\prime}} \in H_{0}$ and $x_{\pi} \in H-H_{0}$. Thus, $y_{\pi} \in H-H_{0}$; hence, $y \in H-H_{0}$ and $D$ is a union of $\pi$-sections of $H$.

If $S$ is a $\pi$-section of $G$ and $S \cap D \neq \varnothing$, let $x$ and $y \in S \cap D$. Then $x$ and $y \in H-H_{0}$, and in particular, $x_{\pi}$ and $y_{\pi} \in H-H_{0}$ with $x_{\pi}$ and $y_{\pi} G$-conjugate. Using condition (A), we see that $x_{\pi}$ and $y_{\pi}$ are $H$-conjugate; hence, $x$ and $y$ lie in the same $\pi$-section of $H$. Therefore, $S \cap D=\varnothing$ or $S \cap D$ is a $\pi$-section of $H$. Lemma 3.4 of $[2]$ now implies that

$$
\left|\left(H-H_{0}\right)^{G, \pi}\right|=\left|D^{G, \pi}\right|=(G: H)|D|=(G: H)\left|H-H_{0}\right| .
$$

Thus, $\left(G, H, H_{0}, \pi\right)$ satisfies conditions $\left(\mathrm{B}_{0}\right)$ and $(\mathrm{C})$, and $H / H_{0}$ is solvable. Theorem 2 follows from Theorem 1.

\section{REFERENCES}

1. D. Gorenstein, Finite groups, Harper \& Row, New York, 1968.

2. H. Leonard, On relative normal complements in finite groups (to appear).

3. W. F. Reynolds, Isometries and principal blocks of group characters, Math. Z. 107 (1968), 264-270.

Department of Mathematics, University of Miami, Coral Gables, Florida 33124 